LAWRENCE LIVERMORE NATIONAL LABORATORY
Deployment, Commissioning and Operation of Plasma Electrode Pockels Cells in the National Ignition Facility

P.A. Arnold, C.W. Ollis, A. Hinz, C. Robb, K.A. Primdahl, J.J. Watson, M.D. O'Brien, W.G. Funkhouser, P.J. Biltoft, R.T. Shelton, W.C.

Tapley, and W.J. Dehope

December 1, 2003

Photonics West 2004, San Jose, California, January 24-29, 2004 
This document was prepared as an account of work sponsored by an agency of the United States Government. Neither the United States Government nor the University of California nor any of their employees, makes any warranty, express or implied, or assumes any legal liability or responsibility for the accuracy, completeness, or usefulness of any information, apparatus, product, or process disclosed, or represents that its use would not infringe privately owned rights. Reference herein to any specific commercial product, process, or service by trade name, trademark, manufacturer, or otherwise, does not necessarily constitute or imply its endorsement, recommendation, or favoring by the United States Government or the University of California. The views and opinions of authors expressed herein do not necessarily state or reflect those of the United States Government or the University of California, and shall not be used for advertising or product endorsement purposes. 


\title{
Deployment, commissioning and operation of plasma electrode Pockels cells in the National Ignition Facility
}

\author{
P.A. Arnold, C.W. Ollis, A. Hinz, C. Robb, K.A. Primdahl, J.J. Watson, M.D. O’Brien, \\ W.G. Funkhouser, P.J. Biltoft, R.T. Shelton, W.C. Tapley and W.J. DeHope \\ Lawrence Livermore National Laboratory \\ Livermore CA USA 94550
}

\begin{abstract}
Large aperture Plasma Electrode Pockels Cells (PEPCs) are an enabling technology in the National Ignition Facility (NIF) at the Lawrence Livermore National Laboratory. The Pockels cells allow the NIF laser to take advantage of multipass main amplifier architecture, thus reducing costs and physical size of the facility. Each Pockels cell comprises four $40-\mathrm{cm} \times 40-\mathrm{cm}$ apertures arranged in a 4x1 array. The combination of the Pockels cell and a thin-film polarizer, also configured in a $4 \times 1$ array, forms an optical switch that is key to achieving the required multi-pass operation.
\end{abstract}

The operation of the PEPC is a follows: Before the arrival of the laser pulse, optically transparent, low-density helium plasmas are initiated to serve as electrodes for the KDP crystals mounted in the Pockels cell. During beam propagation through the main laser cavity a longitudinal electric field is impressed on the electro-optic crystals. The polarization of the propagating beams is rotated by $90^{\circ}$ on each of two passes, thereby allowing the beam to be trapped in the main laser amplifier cavity for a total of four passes before being switched out into the cavity spatial filter.

The physics aspects of the PEPC are well documented. Consequently, this paper will emphasize the PEPC subsystem in the context of its role and relevance within the broader NIF laser system, provide a view of the complexity of the subsystem and give an overview of PEPC's interactions with other elements of NIF, including interfaces to the Beamline Infrastructure, the NIF Timing Subsystem, and the Integrated Computer Control System (ICCS); along with dependence on the Optics Production, Transport and Handling (T\&H), and Assembly, Integration and Refurbishment (AIR) and Operations organizations. Further, we will discuss implementation details related to the functional blocks and individual components that comprise PEPC, with particular emphasis on the unique constraints placed on the elements and the attendant engineering solutions. Finally, we describe performance, fabrication and assembly requirements unique to PEPC and the various considerations necessary for successfully commissioning and operation of each PEPC unit. These considerations include, but are not limited to, materials choices, materials preparation and processing (especially cleanliness), inspection, pre- and post-assembly testing.

Keywords: Pockels cell, plasma electrode, electrooptical switch, pulse generator, NIF, National Ignition Facility

\section{INTRODUCTION}

The Plasma Electrode Pockels Cell (PEPC) is a key technology of the National Ignition Facility. Working in conjunction with a thin-film polarizer, PEPC functions as the active element of an optical switch for the main amplifier cavity, allowing optical pulses to be trapped and then released, thus enabling NIF to take advantage of the gain and attendant cost-savings afforded by a four-pass amplifier design.

The details of the genesis, development and prototyping of the PEPC are well documented ${ }^{1-5}$. PEPC has now moved from its laboratory setting to the NIF facility as a fully functional system. As deployed, PEPC is a complex marriage of state-of-the-art optics, low voltage and high voltage electronics, mechanical, gas and vacuum subsystems all under computer control and operated by a single operator from the NIF central control room. 


\section{PLASMA ELECTRODE POCKELS CELL: CELL OPERATION AND PHYSICAL DESCRIPTION}

\subsection{Pockels Cell operation in NIF}

PEPC's location in a NIF beamline is represented schematically in Figure 1. The beamline depicted in the figure represents but one of the 192 beams that will eventually become operational in NIF.

Referring again to Figure 1, the path of the laser light through the optical system is as follows: A low power optical pulse is injected into the power amplifier at the transport spatial filter, making a gain pass through the power amplifier before entering the periscope, the transition zone between the power amplifier and the main amplifier. Inside the periscope light reflects off laser mirror 3, LM3, and the thin-film polarizer. It then passes through the Pockels cell to enter the slabs of the main amplifier cavity. During the initial pass the Pockels cell is OFF and the polarization of the beam is unaffected. Light reflects off LM1 and returns to the Pockels cell. At this point the Pockels cell is ON. The polarization of the beam is rotated by $90^{\circ}$, allowing the beam to pass through the polarizer and propagate to Laser Mirror 2, LM2. The beam again passes through the polarizer and the Pockels cell. The cell is still ON for the third pass and the polarization of the beam is once again rotated by $90^{\circ}$. Reflecting off LM1 the beam begins its fourth pass through the amplifier. The Pockels is OFF during pass 4. Light reflects off the polarizer and out of the main amplifier. Thus, by taking advantage of the two $90^{\circ}$ polarization rotations afforded by the Pockels cell and the polarization-dependent properties of the polarizer, the trapped beam completes four gain passes of the 11-slab main amplifier before being switched out of the cavity to once again enter the power amplifier and then propagate to the target chamber.

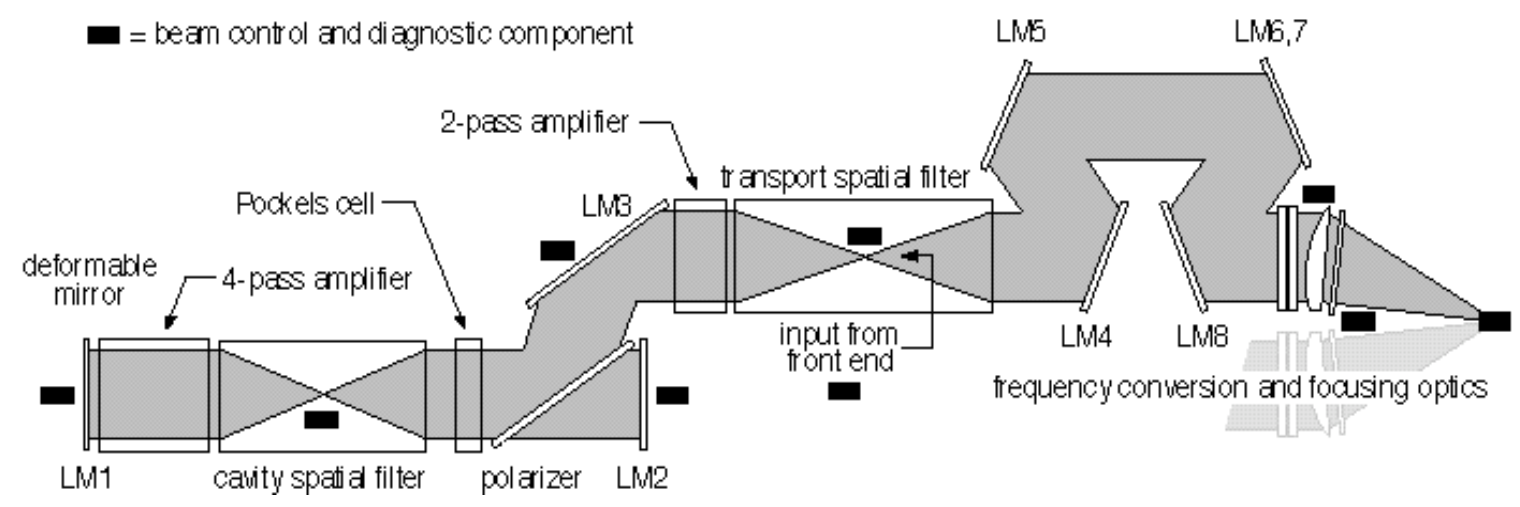

Figure 1. Illustration of the NIF beamline and the relative location of the plasma electrode Pockels cell.

As indicated, the Pockels cell interacts with the beam four-pass traversal of the cavity of the main amplifier. Referring now to the diagram of Figure 2 the detailed sequence of events within the Pockels cell is as follows:

Prior to laser shot operations, the plasma electrode Pockels cell lies in a quiescent state. The regions on either side of the crystal are evacuated to below $1 \times 10^{-5}$ Torr by an on-board turbomolecular drag pump, backed by a mechanical pump. Minutes before the shot, a He- $\mathrm{O}_{2}$ gas mixture is flowed into and through the cell, raising the pressure to $\sim 70$ millitorr in the gas cells on both sides of the crystals.

Approximately 300 milliseconds before the arrival of the laser beam in the cavity, low voltage, low current power supplies excite the low-pressure $\mathrm{He} / \mathrm{O}_{2}$ mixture now residing on both sides of the KDP crystal. The excitation voltage initially exceeds $1 \mathrm{kV}$ but drops quickly to a steady state value of $\sim 300 \mathrm{~V}$ once a gas breakdown is sensed. This has the effect of initiating and then maintaining glow discharges in the gas cells in anticipation of the main plasma discharge.

Ten microseconds before the beam arrives the electron density of the plasma in each gas cell is raised to approximately $10^{12} \mathrm{~cm}^{-3}$ by applying a multi-kiloampere current, resulting from the discharge of a high voltage energy storage capacitor 
in the cell's Plasma Pulse Generator (PPG). Each plasma region acts as an optically transparent, equipotential surface on the face of the crystal.

Meanwhile, the linearly polarized optical pulse propagates to the cavity polarizer. The s-plane polarization of the beam is such that the light reflects off of the polarizer and into the Pockels cell. The cell is still OFF at this point (that is, no longitudinal electric field has been applied to the KDP though, as noted, plasma electrodes have already been created on

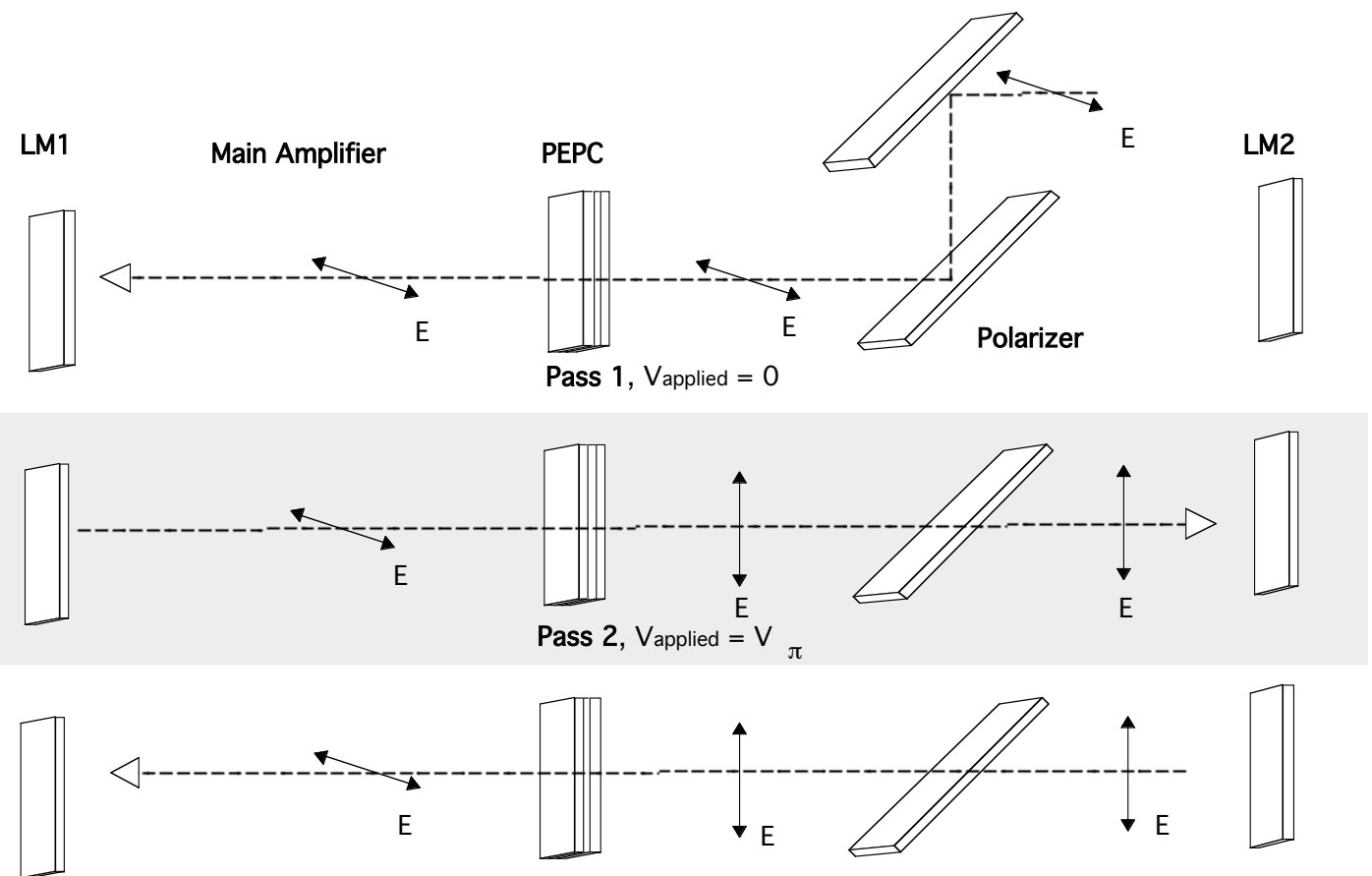

Pass 3, Vapplied $=\mathrm{V}$

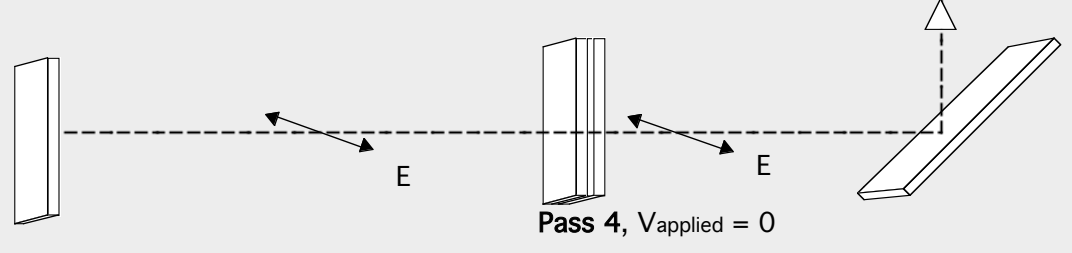

Figure 2. Schematic representation of PEPC operation in NIF.

both sides of crystal). The beam traverses the cell essentially unperturbed (except for absorption of the crystal and small reflections at each optical surface) and continues on its path to the far end of the amplifier, completing Pass 1.

Reflecting off LM1 (laser mirror 1), the beam begins Pass 2.

Triggered so the rising edge of the output pulse of the cell's Switch Pulse Generator (SPG) arrives immediately after the beam has cleared the cell on Pass 1, this high voltage pulse reaches its flattop value in approximately $100 \mathrm{~ns}$. The Pockels cell is now in its $\mathrm{ON}$ state with $\mathrm{V}_{\mathrm{p}}(16.4 \mathrm{kV})$ applied to the crystal, with the applied electric field parallel to the direction of beam propagation. The applied voltage is such that induced birefringence in the crystal rotates the polarization of the beam by $90^{\circ}$. In particular, the amplified beam, having made its second pass through the amplifier laser glass now passes through the active cell and, as described, the polarization rotates as it passes through the KDP crystal. The beam is now p-plane polarized with respect to the polarizer and the beam passes through the polarizer, reflects off LM2, and then passes through the polarizer again. The Pockels cell is still in the ON state as the beam begins Pass 3. The polarization of the light is rotated again by $90^{\circ}$, returning it to the polarization state (s-polarization) of the first pass through the amplifier. 
The beam traverses the main amplifier glass two more times (Passes 3 and 4) and returns yet again to the Pockels cell. By this time, the applied voltage has dropped to zero and the cell is in an OFF state. Thus, the beam passes through the Pockels cell with its polarization unaltered. The s-plane polarized light reflects off the polarizer and back into the 2-pass power amplifier.

It is obvious from this discussion that the Pockels cells must be able to change state from OFF to ON and from ON to OFF in less than the PEPC-to-LM1 cavity roundtrip transit time of approximately $277 \mathrm{~ns}$. This rapid switching allows the injected pulse to be trapped within the cavity on pass 1 and to be switched out after pass 4 . This switching waveform is primarily determined by the use of low impedance pulse forming lines (PFLs) housed in the SPG with additional shaping afforded by a series R-L circuit that is placed in parallel with the capacitance of the cell. The primary effect of the R-L circuit is to decrease the falltime of the pulse and to delay any oscillatory behavior until the fourth pass has cleared the cell.

\subsection{Physical description}

In keeping with the NIF architecture and concept of operation, the Plasma Electrode Pockels Cell is configured as a Line Replaceable Unit (LRU). Fully operational units are delivered to the NIF laser bay to replace units that require maintenance. Refurbishment of the LRU is performed in an off-line, dedicated cleanroom facility. This mode of operation has significant implications for design, with particularly stringent requirements on physical robustness, interchangeability/repeatability and mean-time-between repairs.

The Pockels cell assembly is formed by merging a gas cell (containing the KDP crystals and windows) with a stainless steel frame that provides structural support and interfaces to the NIF Periscope. The fully assembled Pockels cell LRU is $2.6 \mathrm{~m} \times 0.9 \mathrm{~m} \times 0.62 \mathrm{~m}$. Each completed assembly weighs $\sim 800 \mathrm{~kg}$. A kinematic mounting system assures that each aperture of the interchangeable LRUs is positioned with $1 \mathrm{~mm}$ accuracy in the horizontal and vertical axes and has an angular error of less than 250 microradians.

Each NIF Pockels cell LRU comprises a 4x1 vertical array of KDP crystals that switches four independent beams. For each $40 \mathrm{~cm} \times 40 \mathrm{~cm}$ (clear) aperture a non-linear KDP crystal is sandwiched between plenums of low-pressure gas that, when ionized, serve as optically transparent cell electrodes. Sol-gel coated fused silica windows act as the vacuum barrier. KDP crystals are precision-potted into a glass midplane using a space-grade, silicone-based encapsulant. Individual optics are wedged and tilted within the cell to minimize parasitics. The entire cell is given a slight rotation about its vertical axis to steer reflections into beam dumps.

The required lateral dimensions of the KDP, square cross section of the NIF beam, coupled with its fluence and power density eliminate the possibility of using more conventional techniques (e.g., ring electrodes or transparent, conductive thin films) for impressing a uniform electric field on the KDP across the entire face of the crystal. Consequently, plasma electrodes are formed in the low-pressure ( $\sim 70$ millitorr) process gas that resides on both sides of the crystals in each NIF Pockels cell. An electron density of $\sim 10^{12} \mathrm{~cm}^{-3}$ provides sufficient conductivity to produce an equipotential surface, thus allowing a uniform electric field to be applied to the entire KDP crystal, resulting in uniform, efficient switching. ${ }^{4,5}$

Four independent plasma channels exist within the LRU with each spanning a pair of crystals on a given side of the cell centerline. The glass midplane, with its four potted KDP crystals, divides the cell in half along the vertical axis. Four sets of anodes, configured in a centerline assembly, divide the plasma channels along the horizontal axis. Thus, the dimensions plasma channels are approximately $85 \mathrm{~cm}$ x $45 \mathrm{~cm} \times 5 \mathrm{~cm}$.

Figure 3 depicts a majority of the key features of the PEPC LRU, including the frame used to support the unit, the mounting ball which holds the unit in place in the NIF periscope, the $4 \times 1$ array of optical apertures, bifilar chokes that isolate the pulse generators, voltage monitors that provide feedback on the applied crystal bias, high voltage cables that transmit energy to the cells and the baseplate that forms a hermetic seal to the bottom of the pressurize periscope.

Except for the brief periods when the Pockels cell is being used for shot operations, the interior is allowed to pump down to the base pressure of the on-board turbomolecular drag pump. This pressure ranges from the low to mid- $10^{-6}$ Torr, depending on the previous history of the cell. When the cell is going to be operated the process gas is allowed to flow, 
"flooding" the plasma channels and raising the cell pressure to its nominal value of 70 millitorr. Gas flow is limited to a few standard cubic centimeters/minute ( $\mathrm{sccm}$ ). Once a shot has been completed the gas flow is interrupted and the cell is allowed to pump back down to low pressure. A cell unable to reach and maintain a pressure below $5 \times 10^{-5}$ Torr is removed from the beamline for off-line maintenance.

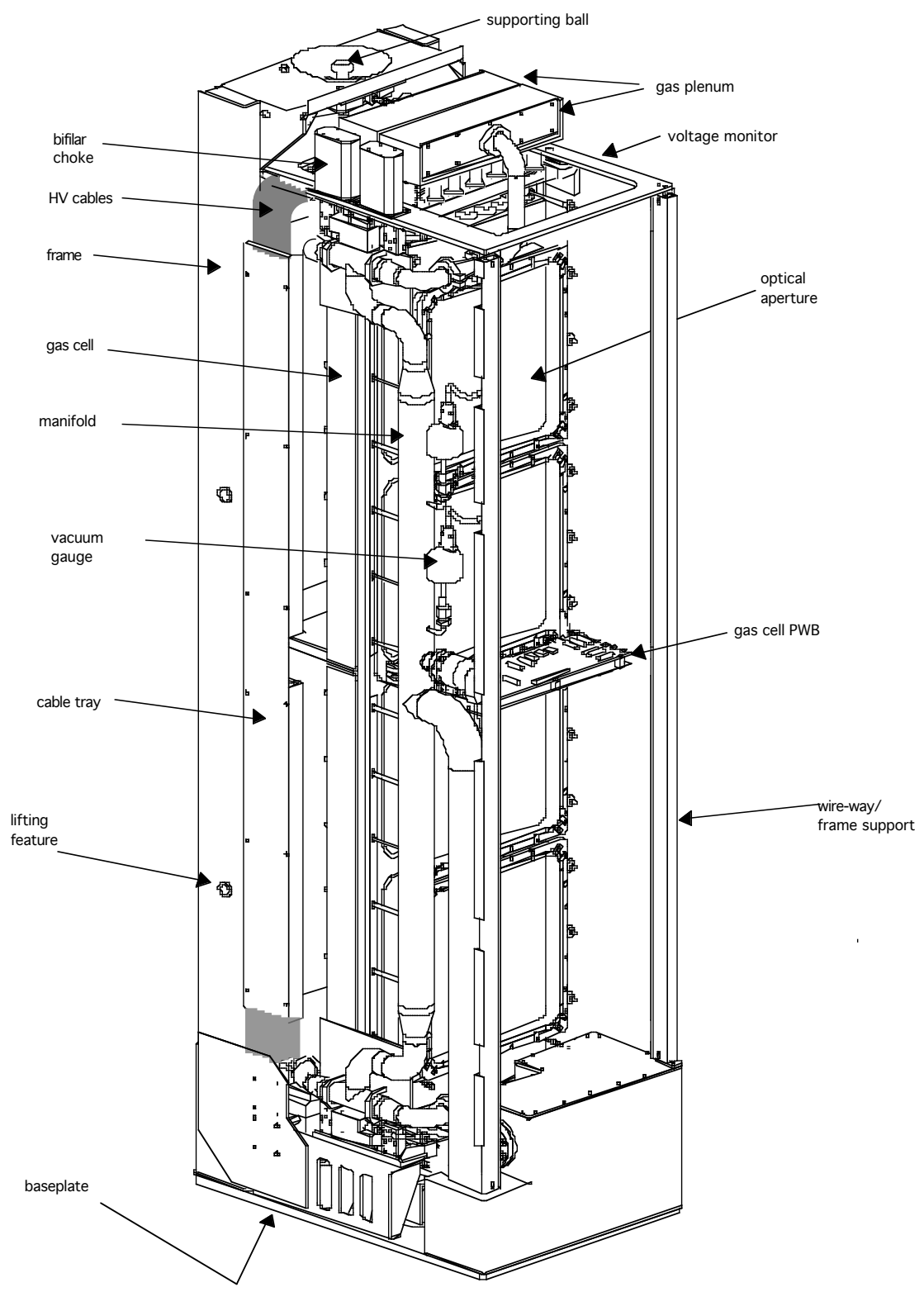

Figure 3. Depiction of the PEPC LRU.

To promote current sharing and plasma uniformity the anode for each gas cell is segmented into 6 individually ballasted electrodes. (See Figure 4.) Cathodes utilize a planar magnetron design to promote ionization and achieve required plasma conductivity and uniformity. Electrodes are recessed to promote uniformity and minimize window and/or crystal contamination. As a further hedge against contamination, electrodes are fabricated from high purity graphite. Ideally, material sputtered from the surfaces of the electrodes oxidizes in the presence of the oxygen in the process gas $(99 \% \mathrm{He}$, $1 \% \mathrm{O}_{2}$ ) and is pumped away as $\mathrm{CO}$ or $\mathrm{CO}_{2}$. 


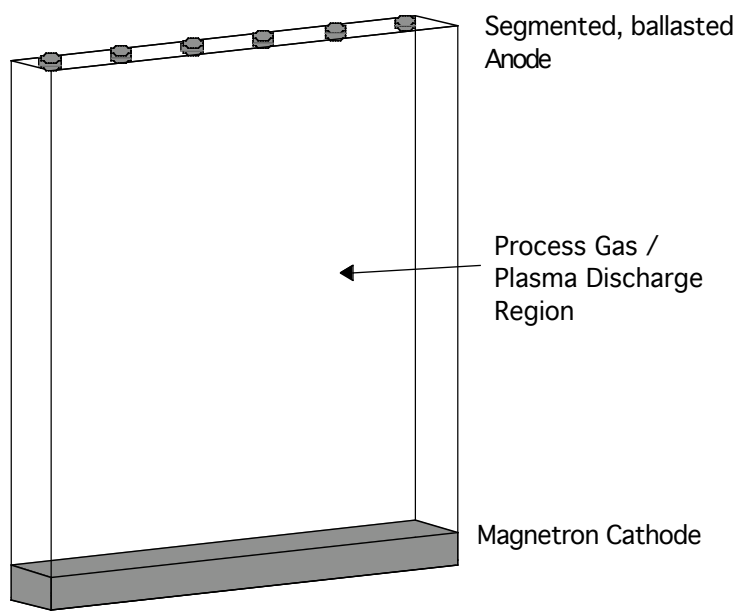

Figure 4. PEPC Electrode Configuration.

The nominally planar plasma in the cells has a tendency to pinch. To overcome this tendency a "magnetic spreading" technique is used to increase plasma uniformity ${ }^{6}$. A substantial fraction of the pulsed discharge current is diverted around the plasma in two identical circuits that route their way around the exterior of the gas cell. Typically, $20 \%$ of the total discharge current is diverted into each of these circuits ${ }^{7}$. The $\mathbf{J} \mathbf{X} \mathbf{B}$ forces resulting from the current flowing through the "spreading circuits" and interacting with the plasma in the cells counteract the pinch effect and lead to a very uniform plasma distribution. Biasing the anodized aluminum cell body to a potential near that of the plasma also enhances plasma performance by imparting a net outward drift to the electron-dominated plasma ${ }^{8}$.

Each Pockels cell is supported by two switch pulse generators (SPGs) and four plasma pulse generators (PPGs). Each PPG contains elements that initiate and then fully develop and maintain the required plasma. The SPGs provide the bias to pairs of KDP crystals in the cell. Simplified schematics of the PPG and SPG appear in Figures 5 and 6, respectively. Both units rely on thyratron main switches. Energy is delivered to the cell via coaxial cables.
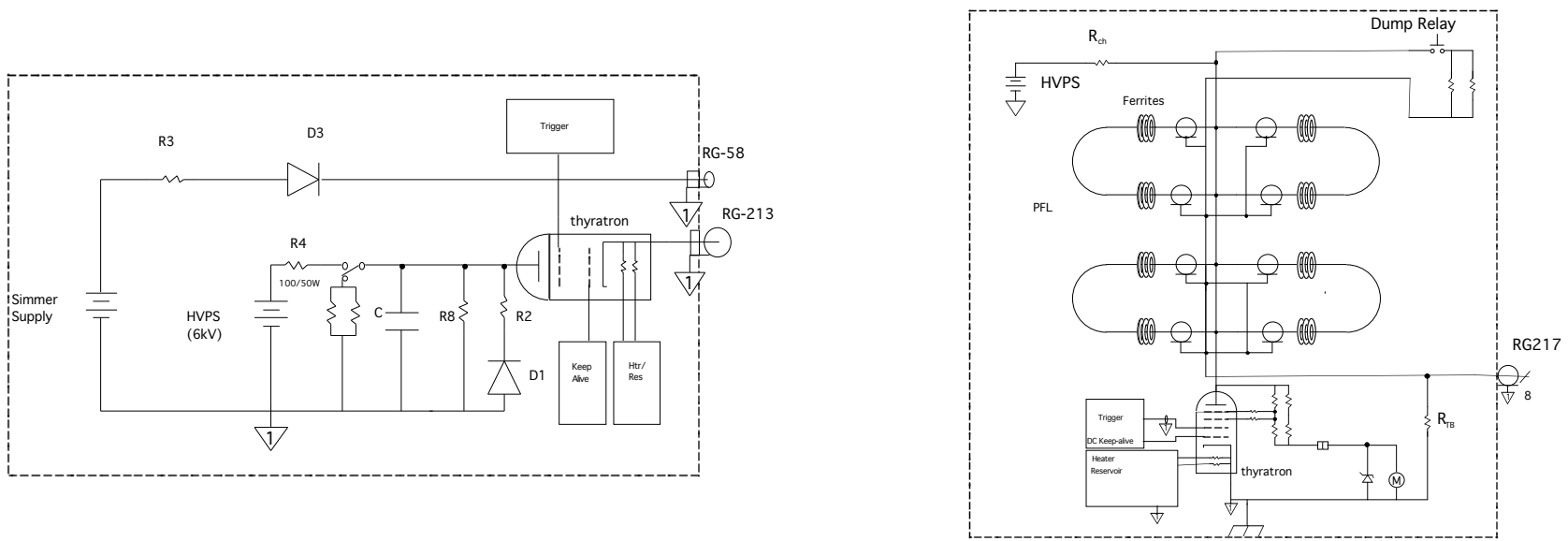

Figure 5. Schematic of PEPC Plasma Pulse Generator (PPG).

Figure 6. Schematic of the PEPC Switch Pulse Generator (SPG).

Low voltage, low current simmer supplies residing in the PPGs create a plasma by initiating a glow discharge in the process gas. This is aided by the existence of auxiliary ("starter") anodes located in close proximity to the planar magnetron cathodes. The short gap between the auxiliary anode and the cathode promotes ready breakdown and 
supplies ions and electrons necessary to create a uniform glow discharge in the larger gas cell. The drive current to the starter anode is heavily ballasted to limit energy delivered to the gap. The conductivity of the plasma is increased to required levels when the main storage capacitors in the PPGs are discharged into the low-pressure gas that has been ionized by the simmer supplies.

The final excitation, produced by the SPG, induces the required birefringence in the KDP and causing the $90^{\circ}$ polarization rotation of the light traversing the cell. The SPGs are low impedance drivers whose output pulses results from switching a $6.25-\Omega$ PFL in the pulser. The plasma on either side of the crystal acts as the electrodes for the uniform application of the electric field.

\section{PEPC: A SYSTEM VIEW}

\subsection{System description and interaction}

PEPC is a complex subsystem and, as such, relies on the contributions of many components. These include not only the Pockels cell itself but also the aforementioned Switch Pulse Generator (SPG), the Plasma Pulse Generator (PPG) along with the various chassis comprising the Controls and Diagnostics (C\&D) Subsystem. Each of these components is designed as a line replaceable unit, i.e., units in the facility are replaced by spares; all repairs take place in an offline facility.

Though only a small portion of the larger NIF laser system, PEPC is nevertheless a significant subsystem in terms of both its complexity and the number of components. When fully deployed, PEPC will include more than 5000 inter-rack cables and a like number of intra-rack cables. Nearly 100,000 meters of high voltage coaxial cable will have been installed. The pulsers and control systems will occupy more than 160 racks. Forty-eight Pockels cells will interact with 192 NIF beams.

PEPC is dependent upon other NIF subsystems. In particular, PEPC is interfaced with and dependent upon the precision NIF timing system and well as Front End Processors (FEPs), data acquisition equipment and computer software/controls provided by the NIF Integrated Computer Control System (ICCS). Java running on PCs provides the presentation (Graphical Users Interface or GUI) layer for operations. Real-time control occurs at the edges of the architecture (in imbedded controllers on the FEPs). Software configuration is table driven as much as possible (utilizing Oracle). The status and control frameworks and applications are coded primarily in Ada, supplemented by Java.

The overall PEPC control system is depicted in block diagram form in Figure 7. The figure also illustrates the relative location and interdependence of the PEPC control system within the global NIF control system. As noted, PEPC is dependent upon the NIF timing system for the precise optical triggers that allow it to effectively interact with the beam as well as capture necessary performance data. It interacts with the system archives to store data from system shots. The PEPC supervisory system is capable of autonomous operation for independent subsystem testing such as that required following installation and during commissioning phases. The supervisory system merges seamlessly with the NIF Shot Supervisory and Shot Director software for integrated, main laser shots.

Even at this early stage of deployment the software is sufficiently well-developed to allow the PEPC subsystem to be controlled by a single operator during shot operations. And while operations and monitoring of all PEPCs in the NIF could be performed by a single operator, it is the ultimate goal of ICCS to achieve completely automated operations of PEPC (and all other) subsystems.

As implemented and installed in NIF, PEPC subsystem hardware occupies a considerable area and is spread over three different levels within the facility, in and around the Periscope structure. PEPC is organized into fundamental units called bundles, with each bundle comprising the equipment required to support eight beams. Thus, each bundle contains 2 Pockels cell LRUs and supporting equipment, including racks that contain requisite pulse generators, front end processors, modems, analog and digital control units (including those required for vacuum /gas systems and pulse generators), signal distribution boxes, data acquisition equipment, etc. The basic bundle architecture will be replicated twenty-four times to support the entire NIF system. This particular architecture is advantageous for simultaneous operation, installation and commissioning exercises that would otherwise be difficult, if not impossible. 


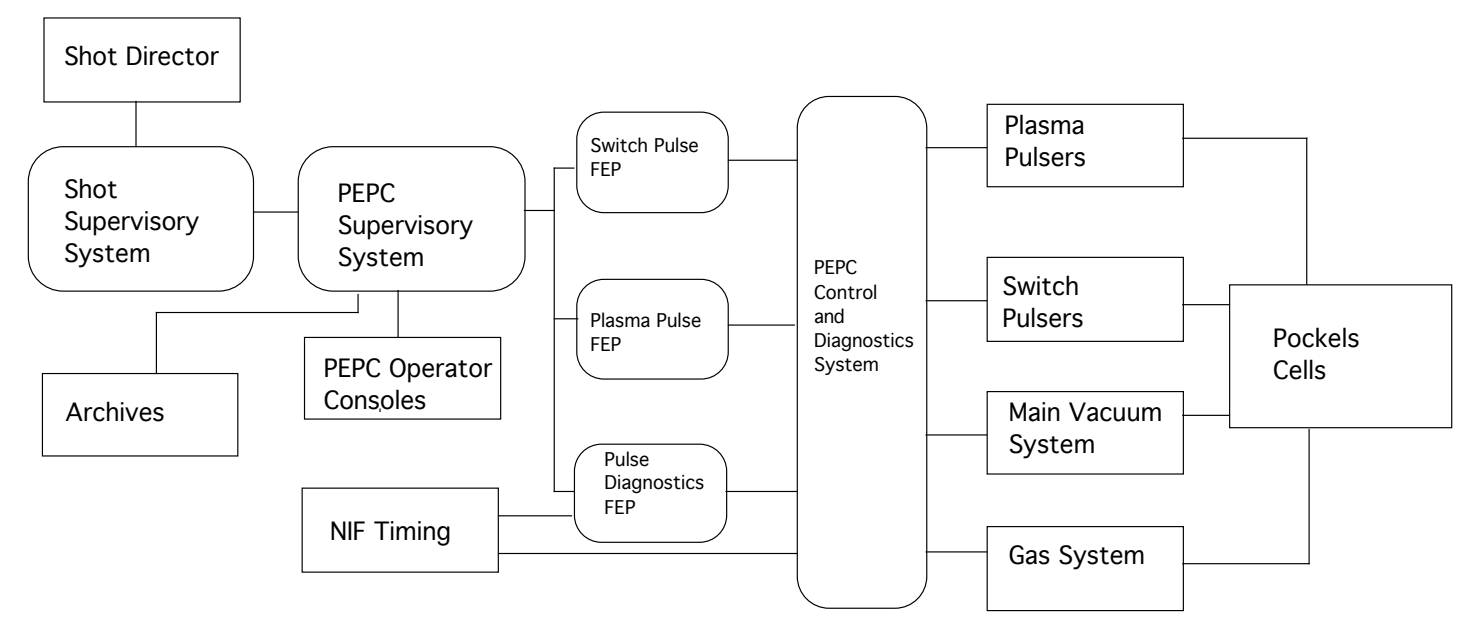

Figure 7. PEPC subsystem as a part of the larger NIF control system.

\subsection{Subsystem shot operations}

While a single PEPC operator participates in a main system shot sequence from the beginning, PEPC operation is essentially limited to the last five minutes of the countdown. In particular, 280 seconds before the main laser is fired, the PEPC operator initiates a sequence of computer-controlled events that culminate in PEPC interaction with the main laser beam to achieve four pass main amplifier performance. At this point gas flow is initiated in the cells, which heretofore are held at the base pressure of the onboard turbomolecular drag pumps. The system waits until pressure stabilizes at 70 millitorr (less than 10 seconds) before starting the low voltage simmer supplies in the plasma pulse generator. Five pulses are applied at the standard PEPC heartbeat rate of $0.2 \mathrm{~Hz}$. At this point the main plasma pulse generator discharges are initiated.

Waveform data for the PPG main current pulses are compared to known standards. Once the PPG waveforms have passed their limit checks the SPGs are started. Once again, the SPG waveforms, corresponding to the applied voltage across the KDP crystals, are automatically checked for performance. SPG temporal alignment is particularly critical. Thus, the control system is capable of temporal resolution of $1 \mathrm{~ns}$. Once all SPGs are determined to be operating normally, flags are set and displayed on the consoles available for viewing by the PEPC operator and the shot director. PEPC continues to fire every 5 seconds as other systems, such as the flashlamp power conditioning system and the preamplifier module power conditioning unit, charge. PEPC continues to trigger until 5 seconds after the main shot, allowing post shot data to be acquired and archived. The computer control system then shuts down PEPC in reverse order, actively monitoring and displaying performance for the operator until the cell pressure has stabilized again below $5 \times 10^{-5}$ Torr.

All operator interaction with the PEPC subsystem is through the computer interface. Automated sequences for shots are initiated and monitored by the operator during normal shot sequences, while manual operation is available for activities such as troubleshooting, installation and commissioning tasks. Color coded status buttons quickly alert the operator to (potential) problems. An automated "drill-down" feature in the software allows the operator to quickly view the failed device and diagnose problems.

PEPC operators required and receive extensive training. Access to the system is defined by an operator's documented understanding of and familiarity with PEPC. Three distinct levels of training for operators as defined by qualification cards maintained under configuration management. 


\section{PEPC STANDARDS FOR DESIGN/FABRICATION/ASSEMBLY/ INSTALLATION AND OPERATION}

\subsection{Beamline standards}

NIF beamline requirements heavily influence virtually every consideration associated with the cells including their basic architecture/design, choice of materials, methods of assembly, installation and operation.

Among the NIF beamline requirements:

- The beamline (in steady state) must meet or exceed the standards of a Class 100 cleanroom. Thus, all components entering the beamline must meet these minimum standards for cleanliness. Transactions must be minimized to keep the beamline clean.

- During a main laser system shot the beamline is flooded with high divergence, high intensity flashlamp light that can volatilize all types of residues, organics, oxides, etc. Thus, certain materials are excluded from the beamline, others are restricted, while others must be shielded from exposure.

- Also during system shots components are subjected to laser light and amplified spontaneous emission (ASE) that can ablate and otherwise degrade surfaces. This places stringent restrictions on materials and their orientations.

- Losses, reflections, ghosts and parasitics within such a system must be well-controlled to maximize performance and to minimize damage of components.

- Line replaceable units that are installed in the beamlines must be interchangeable from form, fit and function standpoints. LRUs must meet minimum standards of performance and fall within the established criteria for dimensional tolerances.

- Reliability, availability and maintainability (RAM) standards are extremely high. Greater than $99 \%$ availability is required.

In response to these requirements, Pockels cells have the following attributes:

Inherent in the PEPC design is a reliance on "clean" materials. To a large extent, the cell is constructed from aluminum and stainless steel. Where stainless steel and aluminum are not usable other clean materials are employed: cables have Teflon jackets and low out-gassing epoxy used for potting inductive isolators (bifilar chokes that prevent SPG pulses from propagating into the PPGs) and voltage monitors. Stainless steel shields are then used to cover these components to protect them from stray light.

Components used in PEPC are machined to very tight tolerances so the final assembly is assured of not only fitting into the beamline but also correctly mating to the three-point kinematic mounting system in the NIF Periscope structure.

PEPC optics are Sol-gel coated to reduce reflections. All optics are wedged. In addition, precision tilts and rotations are incorporated into the assembly.

In-process assembly inspections, post-assembly electrical testing and mechanical checks assure that the Pockels cell meets cleanliness standards, falls within mechanical tolerance allowances, and operates to optical performance guidelines.

\subsection{PEPC assembly/test}

To achieve the necessary cleanliness Pockels cells are assembled at the PEPC workstation in Optics Assembly Building $(\mathrm{OAB})$, adjacent to the main NIF facility. This facility is maintained and operated by the NIF Assembly, Installation and Repair (AIR) organization. The OAB is a class 100 cleanroom used for the final assembly, integration and testing of all NIF optics. All assembly work is performed by workers in "bunny suits." Hoods with facemasks and air circulators are required when optics are being installed or are exposed. 
The majority of the PEPC parts, stored in the NIF warehouse, arrive at the OAB precision cleaned and triple bagged. Large individual parts such as the PEPC Frame are gross cleaned when they arrive from the manufacturer. These parts are then cleaned again in the OAB's precision cleaning facility before making their way to the PEPC workstation to be incorporated into an assembly.

Essentially no lubricants can be used in the assembly of a Pockels cell. One exception is the minimum amount of ultralow vapor pressure grease that is used on o-rings. All stainless steel fasteners are silver-coated to prevent galling. (Experience has shown that precision cleaning causing galling in materials that are otherwise free from this issue.)

Perhaps the most critical subassembly in the Pockels cell is the midplane. This unit consists of a borosilicate glass slab $(2150 \mathrm{~cm} \times 520 \mathrm{~cm} \times 1.3 \mathrm{~cm})$ with four apertures $(41 \mathrm{~cm}$ x $41 \mathrm{~cm})$ into which Sol-gel coated KDP switch crystals are potted using a space-grade encapsulant. Translational and rotational orientations of the crystals with respect to the midplane are very tightly controlled. This subassembly is completed in the Optics Processing Lab (OPL). The midplane is delivered to the $\mathrm{OAB}$ in a special carrying case equipped with a system that provides a flowing gas atmosphere.

The PEPC workstation is equipped with a number of specialized robotic assembly fixtures. The ErgoTech, shown in Figure 8 , is used to bring the halves of the gas cell together with a midplane to form the gas cell assembly. In addition, the ErgoTech is used in conjunction with the NOID (New Optics Insertion Device: a robotic arm) and Vacuum Window Chuck (a vacuum chuck capable of lifting a single PEPC window) to insert windows into the housing. Figure 9 depicts the insertion of a window into its housing. The cell housing assembly is subsequently installed in the cell frame using a Large Area Transporter (LAT). The cell frame is located on a mobile Rail Carriage when the housing is installed. This rail-mounted carriage allows the Pockels cell to be easily moved between the robots used during assembly and the area used for electrical and optical performance inspections. All robotic and transportation devices have been documented in mechanical engineering safety notes (MESNs) and evaluated for particle generation prior to the start of assembly/integration operations.

Inside the OAB the cell/frame assembly makes two trips to Laser Alignment Verification Station (LAVS): one for adjusting the height of the cell within the frame and verifying the orientation of the crystals and windows, the other for setting a 8 milliradian rotation of the cell about the vertical $(\mathrm{Y})$ axis. (This adjustment steers reflections into beam dumps in the laser optical system.) As suggested by its name, LAVS uses a low power laser to determine the orientation and position of optics. This precision tool is capable of resolving the 12 reflections generated by the two windows and crystal in each PEPC aperture.

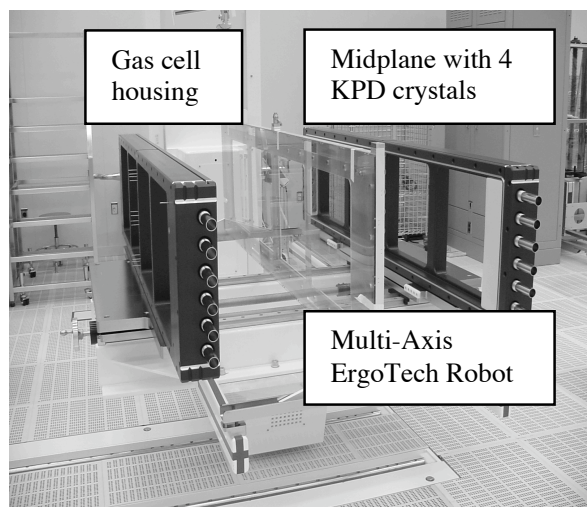

Figure 8. PEPC cell housing and midplane mounted on ErgoTech.

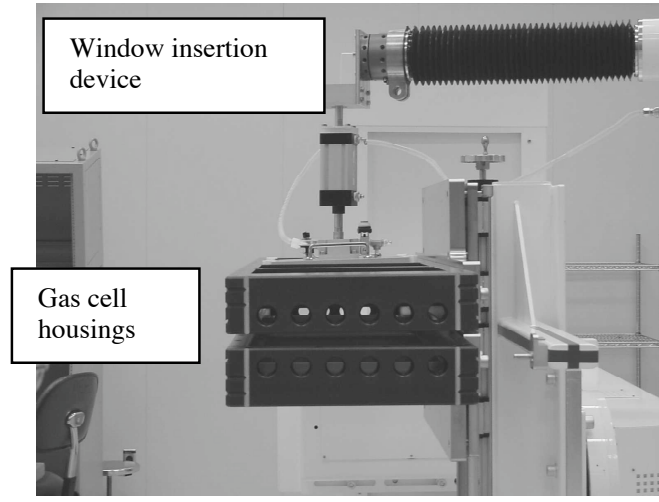

Figure 9. Insertion of Window into Gas Cell using vacuum chuck mounted on NOID.

A fully functional electrical system deployed in the Optics Assembly Building is used to provide assurance that Pockels cells built in the OAB are properly assembled and can be expected to perform to NIF standards when installed in the Laser and Target Area Building (LTAB). Consequently, a Pockels cell, built in accordance with the PEPC LRU Assembly Procedure, is evacuated, backfilled with process gas and then energized by the 4 PPGs and 2 SPGs. The cell 
is tested under normal operating conditions to determine if an assembly meets minimum standards. In order to diagnose the LRU, the plasma in each of the four apertures is imaged by a gated camera system (through an optical filter) to check for uniformity and luminous intensity of the plasma on helium emission line at $442 \mathrm{~nm}$. In addition, the Switch pulse voltages and Plasma pulse currents are measured, recorded and compared to nominal values.

Once the Pockels cell has passed its electrical tests it moves to the OAB Cleanliness Verification System. There the unit is evaluated for cleanliness using swipes and bright light sources. This is followed by final visual inspection for overall cell alignment, dimensional compliance and beamline compatibility is then performed. All data is then collected and reviewed by committee. Only those LRUs meeting all requirements are allowed to proceed to the LTAB.

Figures 10 and 11 show a PEPC LRU during the discharge phase associated with the simmer supply and the main current discharge, respectively. Note the simmer phase is characterized by pinching of the plasma whereas the main discharge is much more uniform due to the use of the spreading circuitry discussed above. Careful examination of Figure 11 reveals the current sharing among the segmented anodes (in the vertical center of the cell) during the multikiloampere main plasma discharge.

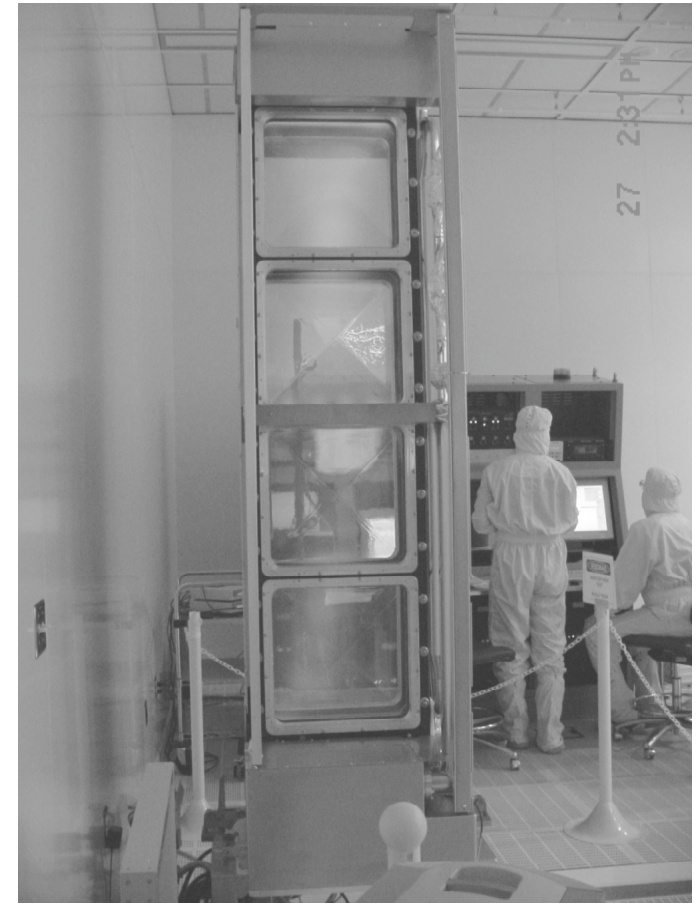

Figure 10. Simmer discharge in the Pockels cell. Optics are protected by special low outgassing, plastic covers.

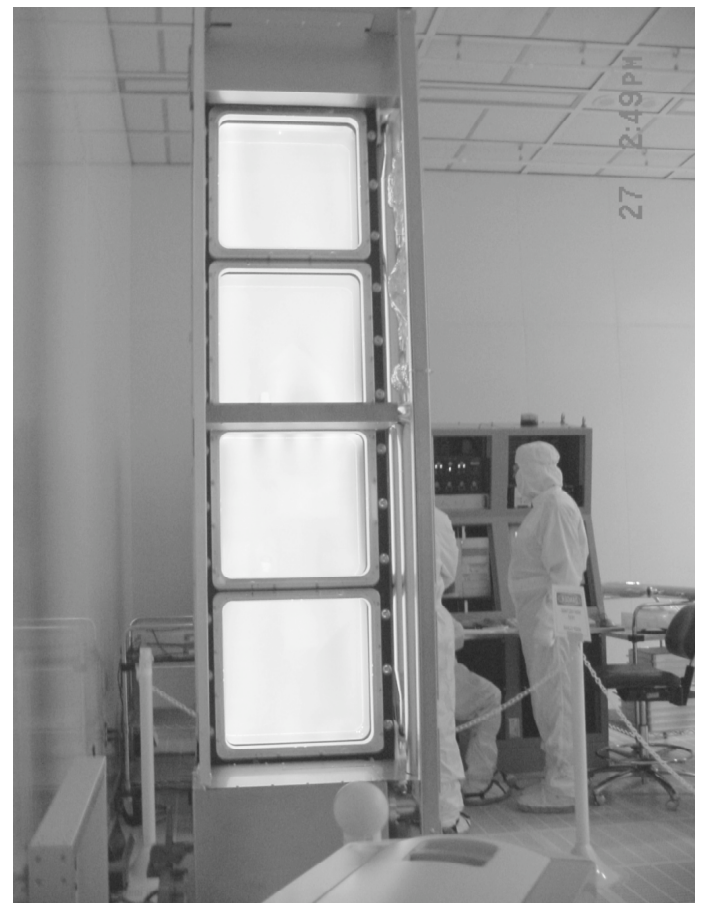

Figure. 11. Main plasma discharge in the Pockels cell.

Pockels cells are transported to the NIF periscope on a semi-autonomous vehicle equipped with a canister that fully encloses the cell. Once the transporter has made its way to the periscope, the canister, essentially a mobile cleanroom, then mates to bottom of the beamline. Covers then swing out of the way and the cell is lifted into its kinematic mounting system. At this point engagement is verified, the clamping system cycled and covers re-close, sealing the beamline. At this point the cell is hidden from view. Problems must be inferred. Primary diagnostics include electrical waveforms and vacuum system performance. 


\section{PEPC PERFORMANCE TO DATE}

Installation of PEPC equipment in the National Ignition Facility began in the summer of 2002. A pair of Pockels cells was installed, along with all necessary ancillary equipment e.g., 4 SPGs, 8 PPGs and more than 30 individual control and diagnostics chassis occupying 12 racks all tied together by several hundred cables. Prior to installation a fully-functional offline facility tested hardware designs and software control-- including multi-cell simulations ${ }^{7}$.

Initial operation of the Pockels cell was limited to the bottom two apertures of a single LRU. Soon a second pair of beamlines was commissioned. Standard operation since the fall of 2002 has been four beams relying on pairs of bottom apertures to support single "quad" (2 x 2 aperture) operation.

Pockels cells have been extremely reliable. One of the cells has operated in the NIF beamline for well over a year. The other installed Pockels cell was removed briefly to fix a small vacuum leak. Its total installation time is currently approaching a year. No pulsers have failed; the controls and diagnostics system has experienced no chassis failures. Multiple operators from the NIF Operations organization have been trained during NEL to support two-shift operations.

\section{FUTURE}

Over the next 5 years a total of 48 Pockels cells and their ancillary equipment will be fabricated, assembled, installed and commissioned. All equipment will be built from drawings under configuration management. External vendors will be relied on to supply custom parts and assemblies. While Pockels cells will continue to be integrated at Lawrence Livermore, all other assemblies will arrive fully tested and ready for installation and commissioning.

\section{REFERENCES}

1. J. Goldhar and M.A. Henesian, "Electro-optical switches with plasma electrodes," Opt. Lett., vol.9, pp. 73-75, 1984.

2. J. Goldhar and M.A. Henesian, "Large-aperture electrooptical switches with plasma electrodes," IEEE J. Quantum Electron., vol. QE-22, pp. 1137-1147, 1986.

3. M.A. Rhodes, B. Woods, J.J. DeYoreo, D. Roberts, and L.J. Atherton, "Performance of large-aperture optical switches for high-energy ICF lasers," Appl. Opt., vol. 34, pp. 5312-5325, 1995.

4. C.D. Boley and M.A. Rhodes, "Modeling of plasma behavior in a plasma electrode Pockels Cell," IEEE Trans. Plasma Sci., vol. 27, pp. 713-726, June 1999.

5. M.A. Rhodes, S. Fochs, P. Biltoft, T. Alger, B. Funkhouser, C.D. Boley, C. Ollis, and C. Robb, "4x1 PEPC Operational Prototype," NIF Document 0012961, August 1998.

6. S.N. Fochs, M. Rhodes and C. Boley, "B-Field interactions and electrode optimization the plasma electrode Pockels cell, 2nd Ann. Int. Conf. Solid State Lasers for Application to ICF, 1996, SPIE vol. 3047, pp. 680-691.

7. B. DeHope, A. Hinz, and C. Ollis, "Investigation of magnetic interactions among NIF PEPCs", NIF Internal Memo, April 5, 2002.

8. M.A Rhodes, internal communication.

This work was performed under the auspices of the U.S. Department of Energy by University of California, Lawrence Livermore National Laboratory under contract No. W-7405-Eng-48. 